\section{Co-occurrence of cohesin complex and Ras signaling mutations during progression from myelodysplastic syndromes to secondary acute myeloid leukemia}

\begin{abstract}
Marta Martín-Izquierdo, ${ }^{1 *}$ María Abáigar, ${ }^{1 *}$ Jesús M. Hernández-Sánchez, ${ }^{1}$ David Tamborero, ${ }^{2,3}$ Félix López-Cadenas, ${ }^{4}$ Fernando Ramos, ${ }^{5}$ Eva Lumbreras, ${ }^{1}$ Andrés Madinaveitia-Ochoa, ${ }^{6}$ Marta Megido, ${ }^{7}$ Jorge Labrador, ${ }^{8}$ Javier SánchezReal, ${ }^{5}$ Carmen Olivier, ${ }^{9}$ Julio Dávila, ${ }^{10}$ Carlos Aguilar, ${ }^{11}$ Juan N. Rodríguez, ${ }^{12}$ Guillermo Martín-Nuñez, ${ }^{13}$ Sandra Santos-Mínguez, ${ }^{1}$ Cristina Miguel-García, ${ }^{1}$ Rocío Benito, ${ }^{1}$ María Díez-Campelo ${ }^{4 *}$ and Jesús M. Hernández-Rivas ${ }^{1,4 \#}$

${ }^{1}$ Institute of Biomedical Research of Salamanca (IBSAL), Cytogenetics-Molecular Genetics in Oncohematology, Cancer Research Center-University of Salamanca (IBMCC, USAL-CSIC), Salamanca, Spain; ${ }^{2}$ Research Program on Biomedical Informatics, Hospital del Mar Medical Research Institute (IMIM), Universitat Pompeu Fabra, Barcelona, Spain; ${ }^{3}$ Department of Oncology-Pathology, Science for Life Laboratory, Karolinska Institutet, Stockholm, Sweden; ${ }^{4}$ University of Salamanca, IBSAL, Hematology, Hospital Clínico Universitario, Salamanca, Spain; ${ }^{5}$ Hematology, Hospital Universitario de León, Institute of Biomedicine (IBIOMED)-University of León, León, Spain; ${ }^{6} \mathrm{Hematology,} \mathrm{Hospital}$ Universitario Miguel Servet, Zaragoza, Spain; ${ }^{7}$ Hematology, Hospital del Bierzo, Ponferrada, León, Spain; ${ }^{8}$ Hematology, Hospital Universitario de Burgos, Burgos, Spain; ${ }^{9}$ Hematology, Hospital General de Segovia, Segovia, Spain; ${ }^{10}$ Hematology, Hospital Nuestra Señora de Sónsoles, Ávila, Spain; ${ }^{11}$ Hematology, Hospital Santa Bárbara, Soria, Spain; ${ }^{12}$ Hematology, Hospital Juan Ramón Jiménez, Huelva, Spain and ${ }^{13}$ Hematology, Hospital Virgen del Puerto, Plasencia, Spain
\end{abstract}

* $M A$ and MMI contributed equally as co-first authors.

${ }^{\#} M D C$ and JMHR contributed equally as co-senior authors.

\section{ABSTRACT}

M yelodysplastic syndromes (MDS) are hematological disorders at high risk of progression to secondary acute myeloid leukemia (sAML). However, the mutational dynamics and clonal evolution underlying disease progression are poorly understood at present. In order to elucidate the mutational dynamics of pathways and genes occurring during the evolution to sAML, next-generation sequencing was performed on 84 serially paired samples of MDS patients who developed sAML (discovery cohort) and 14 paired samples from MDS patients who did not progress to sAML during follow-up (control cohort). Results were validated in an independent series of $388 \mathrm{MDS}$ patients (validation cohort). We used an integrative analysis to identify how mutations, alone or in combination, contribute to leukemic transformation. The study showed that MDS progression to sAML is characterized by greater genomic instability and the presence of several types of mutational dynamics, highlighting increasing (STAG2) and newlyacquired (NRAS and FLT3) mutations. Moreover, we observed co-operation between genes involved in the cohesin and Ras pathways in 15-20\% of MDS patients who evolved to sAML, as well as a high proportion of newly acquired or increasing mutations in the chromatin-modifier genes in MDS patients receiving a disease-modifying therapy before their progression to sAML.

\section{Introduction}

Myelodysplastic syndromes (MDS) are a heterogeneous group of clonal hematopoietic disorders characterized by peripheral blood (PB) cytopenia with dysplastic bone marrow (BM) morphology and an increased risk of progression to secondary acute myeloid leukemia (sAML) ${ }^{1-3}$ Approximately one third of patients diag-
Haematologica 2021

Volume 106(8):2215-2223

\section{Correspondence:}

JESÚS M HERNÁNDEZ-RIVAS

jmhr@usal.es

Received: February 3, 2020.

Accepted: July 14, 2020.

Pre-published: July 16, 2020.

https://doi.org/10.3324/haematol.2020.248807

(C)2021 Ferrata Storti Foundation

Material published in Haematologica is covered by copyright. All rights are reserved to the Ferrata Storti Foundation. Use of published material is allowed under the following terms and conditions:

https://creativecommons.org/licenses/by-nc/4.0/legalcode. Copies of published material are allowed for personal or internal use. Sharing published material for non-commercial purposes is subject to the following conditions:

https://creativecommons.org/licenses/by-nc/4.0/legalcode, sect. 3. Reproducing and sharing published material for commercial purposes is not allowed without permission in writing from the publisher. 
nosed with MDS eventually transform into sAML. ${ }^{4}$ Disease progression is associated with a dismal prognosis, partly because most of these patients are resistant to currently available treatments, and the long-term survival rate of treated patients is less than $10 \%$ after a couple of years. ${ }^{5-7}$

In recent years, new high-throughput genomic technologies, such as next-generation sequencing (NGS), have enabled a large number of studies to elucidate some of the mechanisms involved in MDS pathogenesis such as epigenetic regulation, transcription, signaling pathways, splicing, cohesin complex, apoptosis and angiogenesis. However, MDS exhibit great genetic and clinical heterogeneity, so the nature of their pathogenesis is still not fully understood. ${ }^{8-10}$

The mutational dynamics and clonal evolution underlying disease progression have just begun to become clear. Previous studies have identified multiple genes recurrently mutated in MDS and sAML and these have provided insight into the great intratumoral heterogeneity typical of progression from MDS to sAML.-13 These studies have shown that the evolution of the disease is a complex process involving new additional alterations co-existing with the MDS founder clone. ${ }^{14}$ Moreover, recent studies have described the association of mutations in genes such as TET2, RUNX1, ASXL1, and STAG2 with high-risk MDS, as well as the presence of mutations in genes activating signaling pathways, such as FLT3, PTPN11, NPM1, and NRAS, which are newly acquired in SAML and associated with faster progression..$^{5-17}$ However, this complexity and the lack of large cohorts of serial samples means that molecular mechanisms of disease progression are only partly understood. Thus, longitudinal sequencing genomic studies are still required to determine which mutations or combinations of them are important in leukemic transformation.

In this study, we performed whole-exome sequencing (WES) and/or targeted deep sequencing (TDS) on serial samples from MDS patients who evolved to sAML (discovery cohort) before and after progression, as well as TDS on additional MDS patients who did not progress to sAML during follow-up (control cohort). The results were validated in an independent series of MDS patients (validation cohort). Interestingly, we undertook an integrative analysis to determine the mutational dynamics of the pathways and genes and to identify how mutations, alone or in combination, contribute to leukemic transformation. The study showed involvement of co-occurrence of alterations in the cohesin and Ras pathways in the MDS transformation to sAML, as well as a high proportion of newly acquired or increased clonal selection of mutations in the chromatinmodifier genes in MDS patients who received a diseasemodifying therapy before their progression to sAML.

\section{Methods}

\section{Study design}

In order to study the mutational changes occurring during the evolution to sAML from a previous myelodysplastic phase, 486 samples from 437 patients were included in the study. The patient series was divided into three cohorts (Online Supplementary Figure S1): i) discovery cohort: a cohort of MDS $\rightarrow$ sAML progressing patients that included 42 patients diagnosed with MDS who progressed to sAML; according to the study design, $84 \mathrm{BM}$ serial patient-matched samples were collected and sequenced on two occasions with the first sampling, at initial presentation of the disease (diagnosis, MDS stage), and the second sampling, after pro- gression to sAML (disease evolution, leukemic phase); all samples were analyzed by a TDS strategy; furthermore, 16 of those progressing patients ( 32 samples) were initially studied by WES; information about the treatment received before progression was available for all 42 patients: azacytidine $(n=16)$, lenalidomide $(n=4)$ and no treatment or supportive care $(n=22)$; ii) control cohort: a cohort of MDS non-progressing patients consisted of $14 \mathrm{BM}$ paired samples from seven MDS patients who did not progress to sAML after a minimum of 3-year follow-up for low-risk MDS (LR-MDS) and 1 year for high-risk MDS (HR-MDS) (median follow-up of 52 months; range, 20-89 months); according to the study design, the second sampling in this control cohort corresponded to a time when the disease was stable and TDS was performed on all these samples; iii) validation cohort: a cohort of $388 \mathrm{BM}$ or PB samples from patients suffering MDS at diagnosis and for which only one time-point (sample) was studied by TDS; notably, 63 of these patients eventually evolved to sAML, while 325 had not progressed to sAML after a median follow-up of 19.6 months. The main patient clinical characteristics are summarized in the Online Supplementary Table S1.

This research was performed in accordance with the Declaration of Helsinki guidelines, and was approved by the Local Ethics Committee ("Comité Ético de Investigación Clínica, Hospital Universitario de Salamanca"). All patients provided written informed consent.

\section{Sequencing analysis \\ Whole-exome sequencing}

WES was performed on matched diagnosis-progression samples from 16 patients of the discovery cohort. The mean coverage of WES was 77.6x (range, 36-124) and at least 73\% of the captured regions had a coverage of $30 \mathrm{x}$ or more for all 32 samples (Online Supplementary Table S2). See the Online Supplementary Appendix for full details.

\section{Targeted-deep sequencing}

All genomic DNA samples underwent TDS using an in-house custom capture-enrichment panel of 117 genes previously related to the pathogenesis of myeloid malignancies (Online Supplementary Table S3). The mean coverage of TDS was $665 x$ (range, 251-1,198) where $99.5 \%$ of target regions were captured at a level greater than 100x. See the Online Supplementary Appendix for full details.

\section{Analysis of mutational dynamics}

The main aim of this study was to analyze the mutational changes occurring between the first sampling (MDS stage) and the second sampling (stable disease/sAML stage) in the discovery and control cohorts. To this end, variant allele frequency (VAF) at these two stages were compared using two approaches: i) VAF ratio between second and first sampling, where thresholds of $>1.2$ and $<0.8$ were used to classify mutations as increasing or decreasing, respectively, while ratios between these thresholds were considered to be stable; and ii) Fisher's exact test where values of $P<0.05$ were taken to indicate statistically significant changes during progression.

\section{Results}

\section{Molecular landscape of the progression from myelodysplastic syndromes to secondary acute myeloid \\ leukemia}

In order to characterize the main cohort of the study, the discovery cohort, 16 patients (patients \#27 - \#45) were analyzed by WES at the time of diagnosis and at leukemic 
transformation, as previously explained. After a stringent analysis $^{18}$ as described in the Online Supplementary Appendix, 61 variants were identified as likely somatic mutations: 40 were called driver and 21 were called passenger using the novel bioinformatics tool "Cancer Genome Interpreter"19 (Online Supplementary Table S4). A total of 47 variants in genes known to drive myeloid malignancies were further validated with a true positive rate of $>89 \%$ using TDS and VAF correlation between two platforms was high (Pearson's r=0.90) (Online Supplementary Figure S2). However, the application of TDS revealed that several driver mutations were not detected by WES as they were poor- ly covered and displayed a low VAF. Then, we decided to more comprehensively study disease progression by applying the TDS panel in a larger cohort of serially collected samples.

We performed TDS on these 16 patients of the initial discovery cohort and in additional 26 patients. A total of 159 mutations were identified at diagnosis of the 42 patients (Online Supplementary Table S5). The most recurrently mutated genes were TET2 (14 of 42,33\%), SF3B1 (13 of 42, $31 \%$ ), SRSF2 (ten of 42, 24\%), DNMT3A (10 of 42, 24\%), STAG2 (8 of 42, 19\%), TP53 (8 of 42, 19\%) and ASXL1 (6 of $42,14 \%)$. At the time of the second sampling, the sAML
A

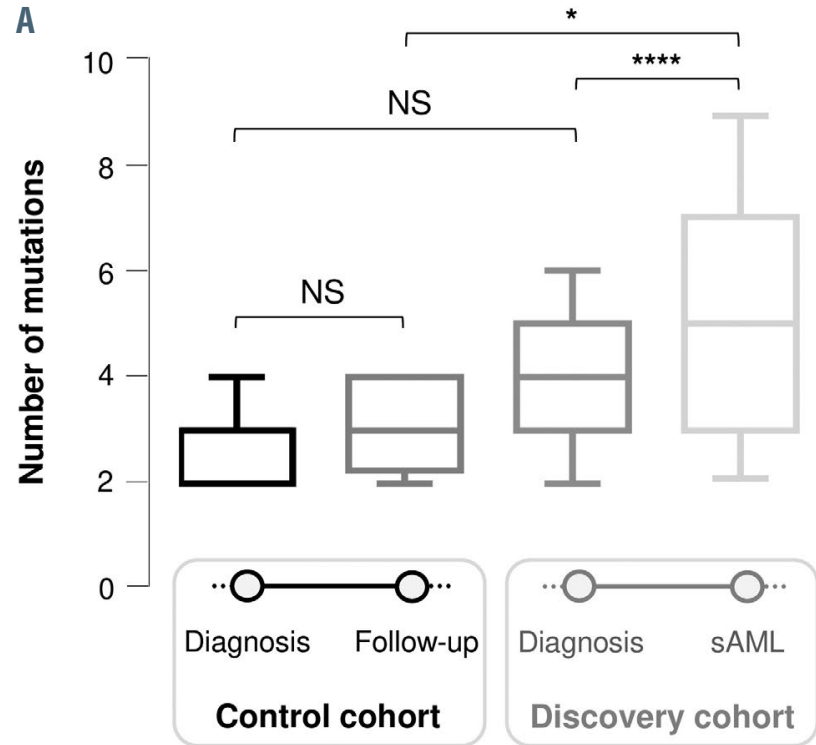

C

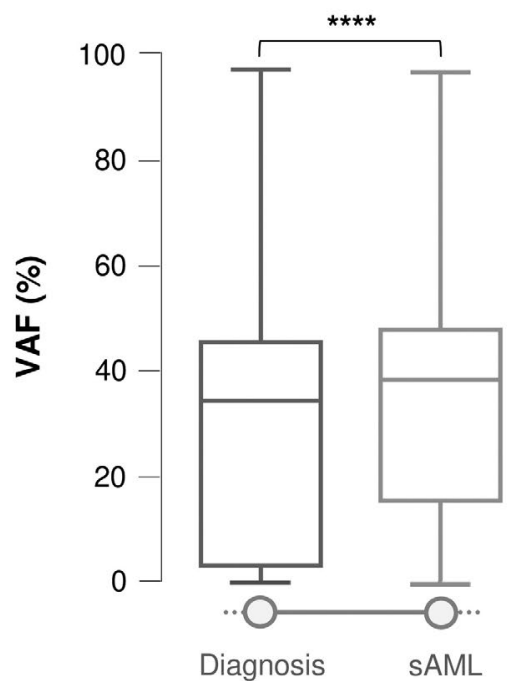

B

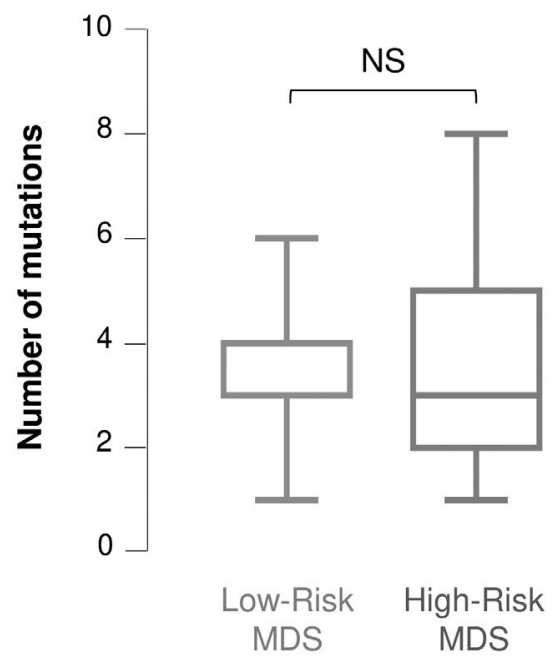

D

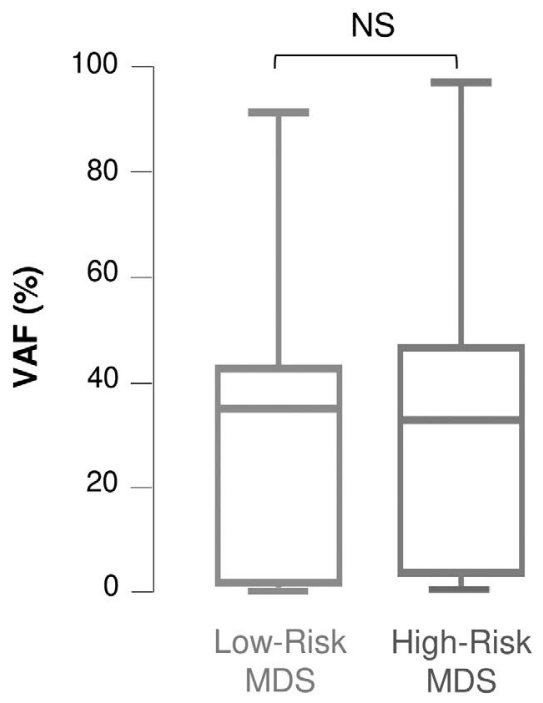

Figure 1. Boxplots showing the differences in the number of mutations (A-B) and variant allele frequency (C-D) between the two times analyzed during the evolution of the disease and between the different French-American-British/World Health Organization (FAB/WHO) subtypes at the time of diagnosis. (A) Differences in the number of mutations between diagnosis and follow-up/secondary acute myeloid leukemia (sAML) stages within and between the control and discovery cohorts. These graphs show a statistically significant increase in the number of mutations during disease evolution in patients who progressed to sAML $(P<0.0001)$. (B) No significant differences in the number of mutations between the FAB/WHO subtypes at time of diagnosis (number of mutations low-risk myelodysplastic syndromes [LR-MDS] vs. high-risk MDS [HR-MDS], $P=0.588$ ). (C) Differences in variant allele frequency (VAF) of detected mutations between diagnosis and sAML stage in the discovery cohort. The VAF was higher at the time of SAML progression $(P<0.0001)$. (D) No significant difference in VAF between the FAB/WHO subtypes at time of diagnosis (VAF: LR-MDS vs. HR MDS, $P=0.528$ ). NS: not significant; $* P<0.05 ; * * * * P<0.0001$. 
A

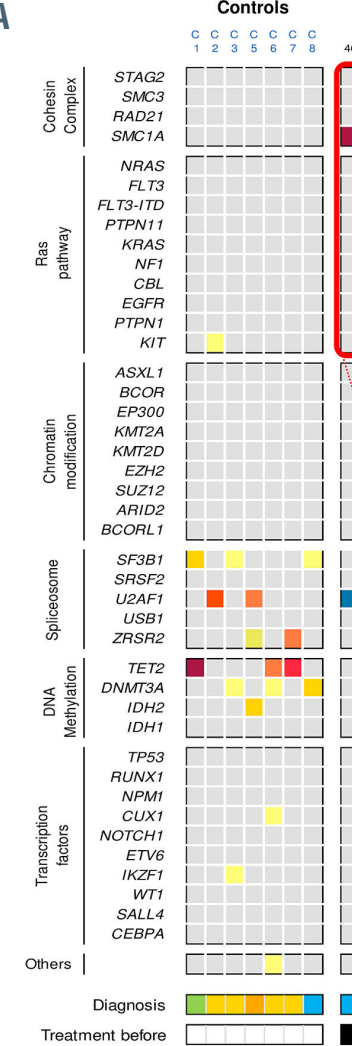

progression
pront
Discovery cohort
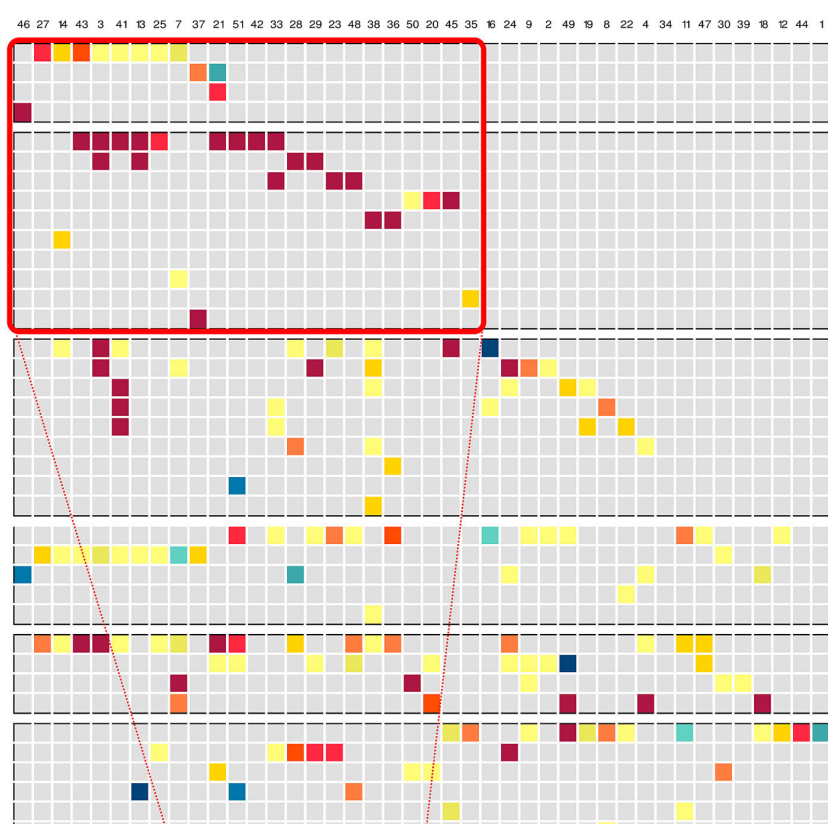

=ニ-
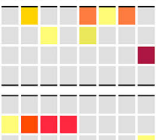

-
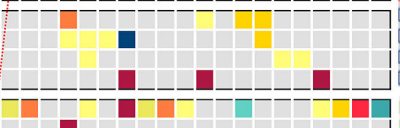

$\square$
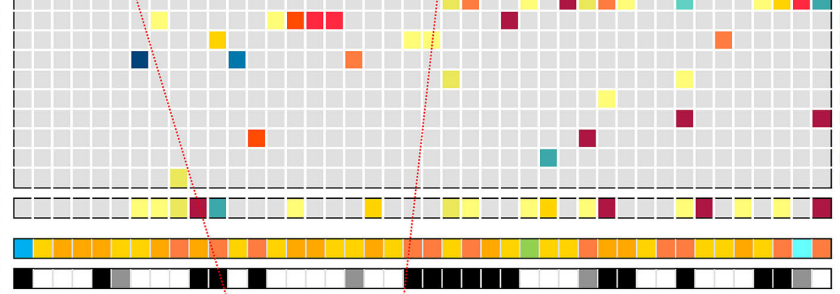

B

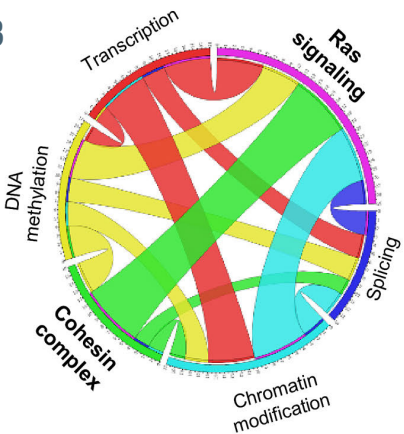

Cohesin complex पागा]

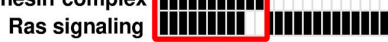

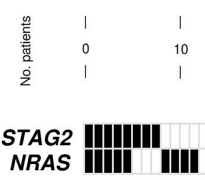

No. mutated patients/gene

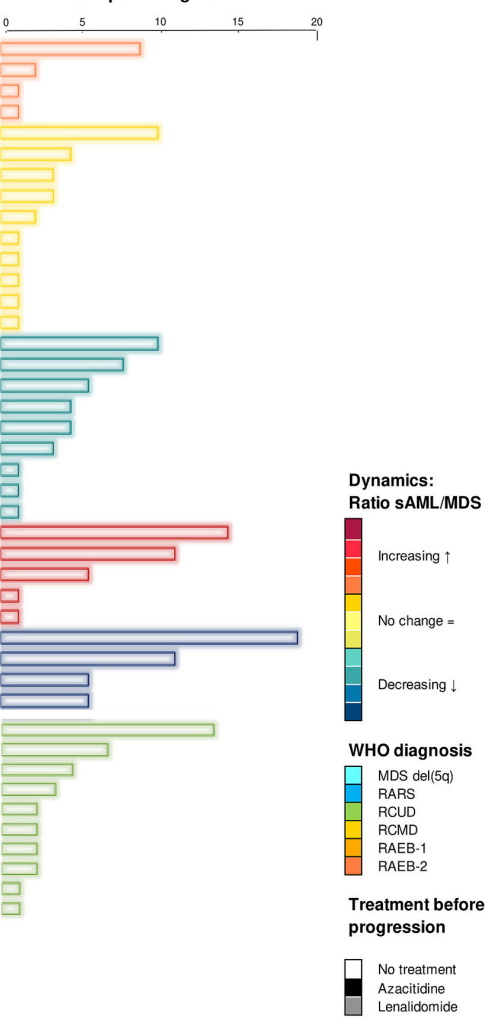

C

\section{Co-occurrence pattern: cohesin complex \& Ras signaling mutations}

Discovery Cohort $(\mathrm{N}=42)$
Validation Cohort $(\mathrm{N}=388)$

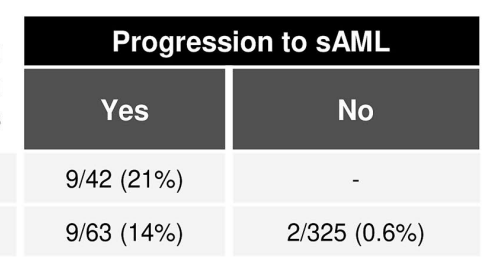

\section{D}
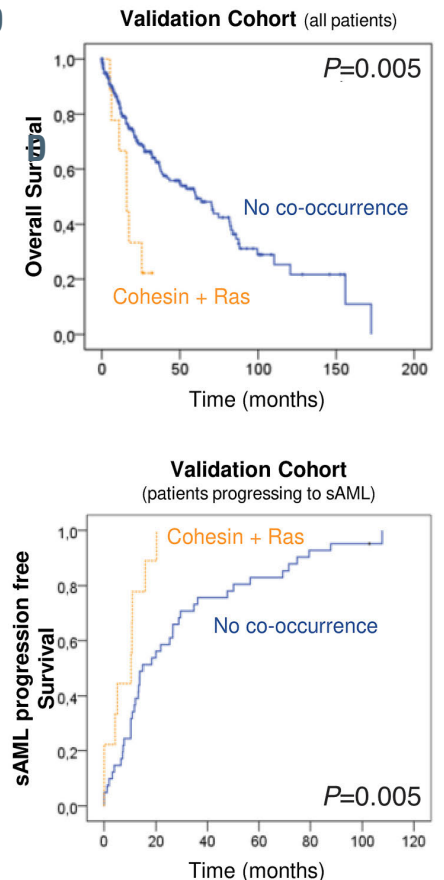

Figure 2. Dynamics of gene mutations in the myelodysplastic syndromes to secondary acute myeloid leukemia progression axis. (A) Comprehensive landscape of mutational dynamics in the discovery and control cohorts. Genes are grouped by cellular functions and are represented in rows; each column represents a patient. Dynamics are represented by a color gradient: red/orange for newly acquired/increasing mutations, yellow for stable mutations, and blue/green colors for decreasing mutations. (B) Co-occurrence of cohesin complex and Ras signaling mutations in the discovery cohort. Circos plot of statistically significant associations between mutations detected in the discovery cohort, grouped by functional pathways. Graphs represent patients with mutations in the cohesin complex and Ras signaling, and the most frequently mutated genes in these pathways, STAG2 and NRAS, showing a statistically significant association ( $P=0.023$ and $P=0.002$, respectively). (C) Incidence of this co-occurrence pattern in the discovery and validation cohorts. The table contains the number of patients with the combination of cohesin and Ras signaling mutations in the discovery and validation cohorts and an indication of whether they evolved to secondary acute myeloid leukemia (sAML). (D) Prognostic impact of the co-occurring mutations in the cohesin complex and Ras pathway. Kaplan-Meier curves for overall survival and sAML progression-free survival in patients bearing co-occurring cohesin and Ras pathway mutations in the entire validation cohort. VAF: variant allele frequency; LR: low-risk; HR: high-risk; NS: not significant; $* P<0.05$. 
stage, 210 mutations were detected, 159 of which were already known to be present in the MDS stage, at clonal or subclonal levels (128 were retained and 31 evolved during disease progression), while 51 were detected only at the second sampling. The most recurrently mutated genes at the sAML stage were similar to those noted at the MDS stage: TET2 (17 of 42, 40\%), SF3B1 (13 of 42, 31\%), TP53 (12 of 42, 29\%), SRSF2 (ten of 42, 24\%), DNMT3A (ten of 42, 24\%), ASXL1 (nine of 42, 21\%) and STAG2 (eight of 42, $19 \%$ ). However, the NRAS gene (9 of 42, 21\%) also stood out at this stage. It should be noted that 32 of 54 genes $(59 \%)$ were mutated only in fewer than three $(<7 \%)$ patients, highlighting the great heterogeneity in the mechanisms of disease evolution.

\section{Regardless of World Health Organization diagnosis subtypes, patients progressing to secondary acute myeloid leukemia present a higher number of mutations than those that do not progress}

In order to analyze the changes in clonal size and distribution during evolution to sAML, we compared the number of mutations identified at diagnosis and at the second sampling in the discovery and control cohorts. The control cohort presented a median of three mutations at both sampling times (p10-p90: 2-4 in both), indicating no significant differences $(P=0.449)$. By contrast, the discovery cohort had a median of four (p10-p90: 1-6) and five (p10-p90: 2-9) mutations at the first and second samplings, respectively, representing a highly significant increase in the number of mutations during disease progression $(P<0.0001)$. Remarkably, the control and discovery cohorts had a similar number of mutations at the time of diagnosis $(P=0.097)$, although a slight trend was observed, while patients who progressed showed a significantly higher number of mutations at the time of sAML than the control patients at the second sampling $(P=0.027)$ (Figure $1 \mathrm{~A})$. Considering the discovery cohort patients by World Health Organization diagnosis subtype (LR-MDS and HR-MDS) did not reveal any significant differences in the number of mutations in patients progressing to sAML $(P=0.588)$ (Figure $1 \mathrm{~B})$.

In order to further study what characterizes disease evolution, we compared the VAF of mutations at both times. Patients who evolved to sAML presented a significantly higher VAF median at second sampling (29.11\% vs. $36.76 \%, P<0.0001$ ) (Figure 1C). However, no differences were identified in the median VAF between each subtype at the time of diagnosis $(P=0.528)$. (Figure 1D).

Therefore, taking all these results together, MDS patients, irrespective of their diagnostic subtype, displayed a greater genomic instability during disease progression than patients who did not evolve to sAML.

\section{Mutational dynamics during the progression to secondary acute myeloid leukemia: clonal evolution}

In order to study the mutational dynamics and identify which mutations could be involved in clonal evolution and play an important role during disease progression, the VAF of mutations detected at both times (followup/sAML vs. diagnosis) were compared in all patients of the discovery and control cohorts.

Four types of clonal dynamics were identified: type 1 , in which mutations were initially present in the MDS stage, but whose VAF increased significantly in the sAML stage; type 2, mutations whose VAF significantly decreased; type 3, mutations that were newly acquired at the sAML stage; type 4, mutations that persisted with a similar allelic burden at both stages.

Stable mutations (Figure 2A, type 4, depicted in yellow) were detected in genes involved in the spliceosome and DNA methylation pathways, such as the splicing factor SRSF2 (diagnosis vs. sAML median VAF, $P=0.4922$ ) and the DNA methylation gene DNMT3A (diagnosis vs. sAML median VAF, $P=0.7695$ ) (Online Supplementary Figure S3).

Only a minority of the mutations detected at diagnosis showed a decrease in their allelic burden (Figure 2A, type

\section{Patient \#43:}

Diagnosis: RAEB-1, $5 \%$ blasts

Time to sAML: 15 months

MDS diagnosis

SAML

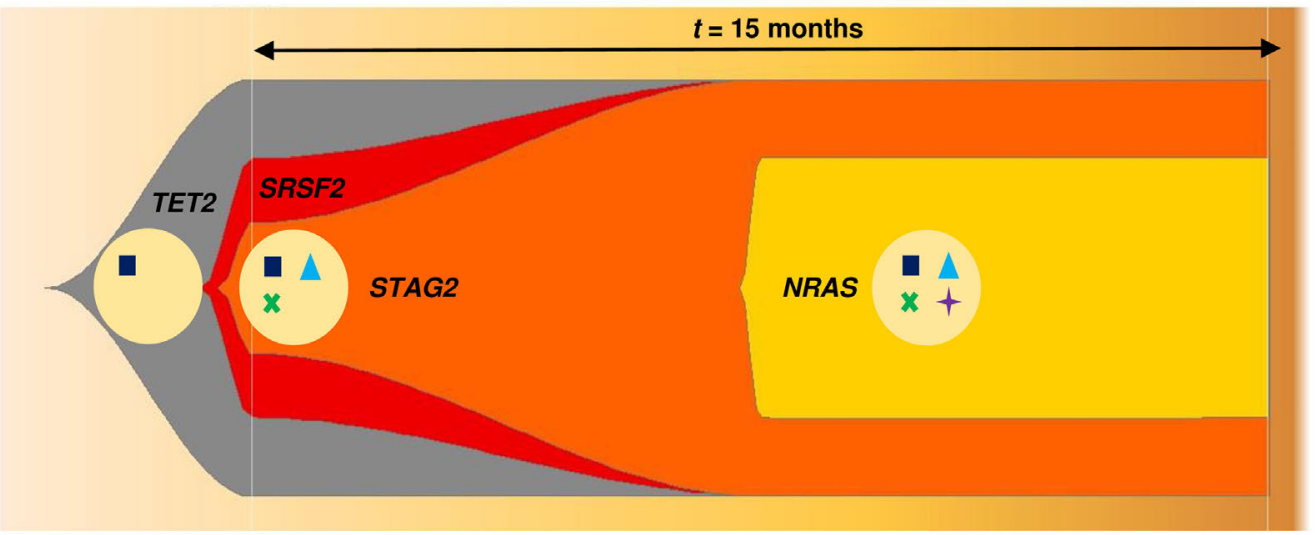

Figure 3. Model of clonal evolution during myelodysplastic syndrome progression to secondary acute myeloid leukemia using patient \#43 as an example and apply ing the Fishplot R package. ${ }^{29}$ In this patient diagnosed as RAEB-1, an myelodysplastic syndrome (MDS) founder clone was present at the time of diagnosis with typical myeloid mutations such as TET2 and SRSF2. This clone also harbored a mutation in STAG2, thus it triggered the acquisition of a subsequent mutation in a Ras pathway gene, namely NRAS. This clone expanded, driving the evolution of the disease. 
2 , in green-blue), and these were randomly distributed throughout all the genes without showing a pattern.

The most interesting dynamic patterns were those of newly acquired mutations (Figure 2A, type 3, represented in red) or increased in clonal size (Figure 2A, type 1, in orange) at the time of sAML progression. These mutational patterns were mainly found in the cohesin complex and Ras signaling, where they were clustered in the STAG2 and NRAS, KRAS and FLT3 genes. These profiles were also detected in transcription factors and epigenetic modifiers, but in these cases they were randomly distributed among the genes. In fact, the STAG2 VAF median was significantly higher at sAML stage (diagnosis vs. sAML median VAF, $P=0.023$ ) and this gene was mutated in eight patients of the discovery cohort, in five of which the VAF had increased by the time they had become sAML (Online Supplementary Figure S3). The increase was statistically significant in three of these five patients, while it was not significant in the other two, although a trend was observed $(P<0.08)$, probably because the VAF at diagnosis was already very high. Moreover, most of the mutations (nine of 12) in the cohesin complex genes were of the frameshift or stop gained (loss of function) type and the cohesin-mutated patients showed a higher number of mutations than wild-type patients (median number of mutations: seven vs. four, $P=0.0179)$. On the other hand, NRAS and FLT3 mutations were newly acquired (diagnosis vs. sAML median VAF, $P=0.0029$ and $P=0.0078$, respectively) during the evolution and so were detected at the sAML stage (Online Supplementary Figure S3).

\section{Co-occurrence of cohesin complex and Ras signaling mutations in patients after progressing to secondary acute myeloid leukemia}

Within this heterogeneous landscape of mutational dynamics, we focused our study on increasing (type 1) and newly acquired (type 3) mutations because their dynamic patterns suggested that they were positively selected during disease evolution. Moreover, in order to better characterize the mechanisms driving sAML progression, we studied which pathways and combination of them were affected by these types of mutations.

In the discovery cohort, a high proportion of Ras signaling-mutated patients at the sAML stage, already harbored cohesin complex mutations. In fact, $26 \%$ (11 of 42) of the discovery cohort patients carried mutations in the cohesin complex at diagnosis. On the other hand, $52 \%$ (22 of 42) of the patients had at least one Ras pathway mutation at the sAML stage, mainly acquired during the evolution of the disease. Of interest, nine of these cohesin-mutated patients (nine of $11,82 \%$ ) carried a cooccurring Ras signaling mutation at the sAML stage. Considering only the most recurrently mutated gene, STAG2 (n=eight of 11), seven patients (seven of eight, $88 \%$ ) carried another mutation in the Ras pathway, this being a NRAS mutation in five patients. Therefore, there was a statistically significant co-occurrence of these two pathways $(P=0.023)$ and of the most recurrently mutated genes of these pathways, STAG2 and NRAS $(P=0.002)$ (Figure 2B).

In order to confirm these observations and their impact on MDS progression to sAML, the combination of the cohesin complex and Ras pathway mutations was sought in the validation cohort, an independent cohort of 388 patients in which the disease was studied on only one occasion, at diagnosis. In fact, these co-occurring mutations were detected in eleven additional patients: nine of which finally transformed into sAML (nine of 63), while two patients did not evolve during the median follow-up of 19.6 months (two of 325) (Figure 2C). Although all samples of this cohort were studied at diagnosis, these nine patients carried cohesin and Ras co-occurring mutations at an advanced stage of the disease, indeed these were detected in sAML sampling or in patients who transformed in a median time of 11 months from sampling.

The discovery cohort included only patients who evolved to SAML, and therefore displayed a very poor outcome. This made it difficult to measure the impact of this co-occurrence in these patients. For that reason and also to further study the clinical consequences of this cooccurrence on outcome, the effects on overall survival and progression-free survival in the validation cohort (median follow-up of 19.6 months) were analyzed. In our validation cohort, where $16.2 \%$ of patients evolved to sAML and $44.76 \%$ died (Online Supplementary Table S1), those patients harboring both the cohesin complex and Ras signaling mutations had significantly shorter overall survival (16 vs. 60 months, $P=0.005$ ) and significantly earlier progression to sAML (10 vs. 15 months, $P=0.005$ ) (Figure 2D). Moreover, in order to study the contribution of the cohesin and Ras mutations alone to these effects, comparison of median overall survival of the doublemutant and cohesin and Ras single mutant patients was performed and patients harboring double mutations showed shorter overall survival than patients with Ras or cohesin single mutations (16 vs. 25 vs. 37 months, respectively, $P=0.018$, Online Supplementary Figure S4).

\section{Higher proportion of newly acquired or increasing mutations in chromatin modifiers in treated myelodysplastic syndrome patients}

As previously mentioned, $48 \%$ of the patients in the discovery cohort of this study were treated with 5-azacytidine (AZA) ( $\mathrm{n}=16)$ or lenalidomide $(\mathrm{n}=4)$, and progressed to sAML after therapy, whereas the other $52 \%$ received no treatment (only supportive care). Thus, we investigated whether the mechanisms of progression could be slightly different between patients who were treated with disease-modifying agents (AZA and lenalidomide) before transformation into sAML and nontreated patients.

In order to achieve this aim, the proportions of the different mutational dynamics were compared between treated and untreated patients. Thereby, the mutational dynamics featured a significantly higher proportion of newly acquired or increasing mutations in chromatin modifiers at the time of sAML in treated patients (eight of 15 mutations), while in untreated patients the majority of mutations were stable (53\% [eight of 15] vs. 19\% [four of 21], $P=0.031$ ). By contrast, and with respect to the treatment, no differences were detected in the dynamics of the cohesin complex $(50 \%$ [three of six] of newly acquired or increasing mutations in treated patients $v s$. $50 \%$ [three of six] in untreated, $P=1.00$ ) or Ras pathway mutations (91\% [ten of 11] in treated vs. 76\% [13 of 17] in untreated patients, $P=0.3299$ ) (Online Supplementary Figure S5). Thus, our study suggests that mutations in chromatin-modifier genes could be related to the evolution of patients who receive disease-modifying treatment before progression to sAML. 


\section{Discussion}

Our study characterizes the landscape of mutations during progression toward sAML and how mutations, alone or in combination, contribute to leukemic transformation, based on the analysis of a large number of serial samples from patients who progressed to sAML. Moreover, the comparison of a control and a validation cohort supports the identification of mechanisms mostly related to evolution to sAML.

Previous studies have documented the clonal evolution and greater clonal heterogeneity during cancer development and, specifically, as MDS evolves to advanced stages and transforms into SAML, ${ }^{8,9,14-16,20}$ and according to this fact, the study demonstrates that MDS patients, irrespective of their diagnostic subtype, gain more mutations and a higher VAF during disease progression. Therefore, these results are evidence that progression toward sAML is associated with pronounced genomic instability and a heavy mutational burden.

The mechanisms of disease evolution showed a great heterogeneity. ${ }^{14-17}$ However, this study identified four distinct types of mutational dynamics and their roles during sAML progression. It is of particular note that this study shows a higher incidence of mutations in the cohesin complex and Ras signaling genes than in previously published MDS series, $8,9,21,22$ probably because this cohort consisted only of patients who progressed to sAML. Moreover, increasing mutations (type 1) were mainly found in genes of this pathway, such as STAG2, most of which were loss of function mutations. Previous studies of cohesin mutations had already shown that these mutations could be related to sAML progression, ${ }^{15,21,22}$ but the dynamics observed in this study confirm that mutations of cohesin complex genes could be an early event in sAML progression, and the loss of function of these genes could play an important role in the leukemic transformation. Similarly, the dynamics of Ras signaling genes observed here, mainly newly acquired mutations (type 3), highlight the importance of this pathway in sAML progression. Earlier studies have described how alterations in Ras pathway genes, such as NRAS and FLT3, could drive the progression to $\mathrm{SAML}^{23-25}$ but these results confirm that they are late events that may drive leukemic transformation. Furthermore, this study reveals a significant cooccurrence of mutations in these two pathways and, also, in the main genes of these pathways (STAG2 and NRAS), excluding the FLT3-ITD mutations which could have a different behavior than other mutations in Ras pathway and they could be involved in an independent mechanism of sAML progression. ${ }^{26,27}$ Although Walter et al. ${ }^{28}$ described that NRAS and cohesin mutations tend to be mutually exclusive, this work included a low number of patients and, conversely, this co-occurrence was briefly described in another study with a higher number of patients. ${ }^{21}$ Thus, this study, due to detecting in a cohort of sAML-progressing patients, demonstrates that this progressive combination of the cohesin complex, mainly STAG2, and Ras signaling mutations, mostly NRAS, could play an important role in the progression of MDS to SAML. In addition, the results from the validation cohort confirmed the impact of this co-occurrence on sAML progression not only in the discovery cohort, but also in the validation cohort. Therefore, these findings support a hypothesis of genetic "predisposition", that early muta- tions shape the future trajectories of clonal evolution from MDS to sAML. Therefore, a new model of genetic evolution could be suggested consisting of cohesin mutations as an early event in the evolution of the disease that trigger to acquire new mutations, mainly Ras signaling mutations. Consequently, this clone expands, driving the disease evolution (Figure 3, model of clonal evolution using Fishplot R package). ${ }^{29}$ Recent studies have described that cohesins are involved in DNA damage repair, chromatin accessibility and transcription factor activity ${ }^{30-32}$ and our results show than cohesin-mutated patients displayed a higher number of mutations, thereby cohesins could cause an instability where new mutations are generated, mainly Ras mutations, leading the disease progression. These are not absolute rules and, unfortunately, this novel model does not fully explain the progression to sAML, but it could explain the evolution in $15-20 \%$ of all sAML transformations. Moreover, cohesin mutations potentiate the subsequent acquisition of Ras mutations, so these mutations could be used to identify patients whose disease is progressing before symptoms associated with progression to sAML are manifested.

On the other hand, the mutations that were stable during the evolution (type 4) were found in splicing and DNA methylation genes. This is in line with the finding of some recent reports showing that variants affect these pathways in this steady-state pattern. ${ }^{16}$ Moreover, mutations in DNA methylation and RNA splicing pathways are well known to have a heavier mutational burden than those in other genes, suggesting an early event in MDS development. 8 Considering these findings together, these results showed that mutations in these pathways, which have high VAF and are stable during the disease evolution, could be directly involved in MDS pathogenesis (driver role) but not in sAML progression (passenger role). The mutations whose VAF decreased during progression (type 2) were distributed randomly throughout all the genes without showing a particular pattern. This could be the result of clone sweeping, a previously described event, ${ }^{15}$ that is specific to each patient rather than to a specific pattern of each gene or pathway.

Furthermore, a mechanism that could be linked to the evolution of patients who receive disease-modifying treatment before progression to sAML was detected. Several studies have described the mutational dynamics in treated MDS patients and have demonstrated that therapy alters clonal distribution, but the predictive impact of the dynamics is still unclear. ${ }^{33-37}$ A significantly higher proportion of newly acquired or increasing mutations in chromatin modifiers at the time of sAML was identified in treated patients. Thus, these results suggest that mutations in chromatin-modifier genes could be related to the evolution of treated patients. However, more studies with larger numbers of patients are required to validate this result.

In summary, MDS progression to sAML is characterized by greater genomic instability, irrespective of the MDS subtypes at diagnosis, and there are four types of mutational dynamics during the disease evolution, increasing and newly acquired mutations (type 1 and type 3 , respectively) being of particular importance. Moreover, a co-occurrence of cohesin complex and Ras signaling mutations could play an important role in the $15-20 \%$ of MDS patients who evolved to sAML. With regard to treatment, we found that mutations in chro- 
matin-modifier genes could be related to the evolution of MDS patients who received disease-modifying treatment before progression to sAML.

\section{Disclosures}

No conflicts of interest to disclose.

\section{Contributions}

MMI designed the experiments, performed targeted-deep sequencing experiments, analyzed the data and wrote the paper; MA designed the experiments, performed whole-exome sequencing experiments, contributed to interpret the results and wrote the paper; JMHS performed NGS data analysis and contributed to the experiment design; $D T$ analyzed the wholeexome sequencing data; $F L C, F R, E L, A M O, M M, J L, J S R$, $C O, J D, C A, J N R$ and GMN provided patient samples and clinical information and SSM and CMG contributed to perform the NGS experiments; RB contributed to data analysis, interpretation of the results and critically reviewed the manuscript and $M D C$ and JMHR conceived the study, designed the experiments and wrote the manuscript. All authors discussed the results and revised the manuscript.

\section{Acknowledgments}

The authors would like to thank to Sara González, Irene Rodríguez, Teresa Prieto, $M^{a}$ Ángeles Ramos, Filomena Corral, $M^{a}$ Almudena Martín, Ana Díaz, Ana Simón, María del Pozo,
Isabel M Isidro, Vanesa Gutiérrez, Sandra Pujante and $M^{a}$ Ángeles Hernández from the Cancer Research Center of Salamanca, Spain, for their technical support. We also thank to Teresa González and Alba Redondo-Guijo for providing patients samples and clinical information and we are deeply grateful to Miguel Quijada Álamo for his helpful suggestions and personal support.

\section{Funding}

This work was supported by grants from the Spanish Fondo de Investigaciones Sanitarias FIS PI18/01500, PI17/01741, Instituto de Salud Carlos III (ISCIII), Fondo de Investigación Sanitaria (Instituto de Salud Carlos III - Contratos Río Hortega (CM17/0017), European Regional Development Fund (ERDF), Una manera de hacer Europa, European Union Seventh Framework Programme [FP7/2007-2013] under Grant Agreement no306242-NGS-PTL, SYNtherapy: Synthetic Lethality for Personalized Therapy-based Stratification in Acute Leukemia (ERAPERMED2018-275); ISCIII (AC18/00093), Proyectos de Investigación del SACYL, Gerencia Regional de Salud de Castilla y León: GRS1850/A18, GRS1653/A17, and Centro de Investigación Biomédica en Red de Cáncer (CIBERONC CB16/12/00233). MMI is supported by a predoctoral grant from the Junta de Castilla y Leon, and by the Fondo Social Europeo (JCYL-EDU/556/2019 PhD scholarship) and JMHS is supported by a research grant from Fundacion Española de Hematología y Hemoterapia.

\section{References}

1. Cazzola M, Della Porta MG, Malcovati L. The genetic basis of myelodysplasia and its clinical relevance. Blood. 2013;122(25):40214034.

2. Lindsley RC, Ebert BL. Molecular pathophysiology of myelodysplastic syndromes. Annu Rev Pathol. 2013;8:21-47.

3. Tefferi A, Vardiman JW. Classification and diagnosis of myeloproliferative neoplasms: the 2008 World Health Organization criteria and point-of-care diagnostic algorithms. Leukemia. 2008;22(1):14-22.

4. Greenberg PL, Tuechler H, Schanz J, et al. Revised international prognostic scoring system for myelodysplastic syndromes. Blood. 2012;120(12):2454-2465.

5. Shukron O, Vainstein V, Kundgen A, Germing U, Agur Z. Analyzing transformation of myelodysplastic syndrome to secondary acute myeloid leukemia using a large patient database. Am J Hematol. 2012 87(9):853-860.

6. Montalban-Bravo G, Garcia-Manero G. Myelodysplastic syndromes: 2018 update on diagnosis, risk-stratification and management. Am J Hematol. 2018;93(1):129-147.

7. Steensma DP, Bennett JM. The myelodysplastic syndromes: diagnosis and treatment. Mayo Clin Proc. 2006;81(1):104-130.

8. Papaemmanuil E, Gerstung M, Malcovati L, et al. Clinical and biological implications of driver mutations in myelodysplastic syndromes. Blood. 2013;122(22):3616-3627.

9. Haferlach T, Nagata Y, Grossmann V, et al. Landscape of genetic lesions in 944 patients with myelodysplastic syndromes. Leukemia. 2014;28(2):241-247.

10. Abaigar M, Robledo C, Benito $\mathrm{R}$, et al. Chromothripsis is a recurrent genomic abnormality in high-risk myelodysplastic syndromes. PLoS One. 2016; 11(10): e0164370.
11. Lindsley RC, Mar BG, Mazzola E, et al. Acute myeloid leukemia ontogeny is defined by distinct somatic mutations. Blood. 2015; 125(9):1367-1376

12. da Silva-Coelho P, Kroeze LI, Yoshida K, et al. Clonal evolution in myelodysplastic syndromes. Nat Commun. 2017;8:15099.

13. Sperling AS, Gibson CJ, Ebert BL. The genetics of myelodysplastic syndrome: from clonal haematopoiesis to secondary leukaemia. Nat Rev Cancer. 2017;17(1):5-19.

14. Walter MJ, Shen D, Ding L, et al. Clonal architecture of secondary acute myeloid leukemia. N Engl J Med. 2012;366(12):10901098.

15. Makishima $\mathrm{H}$, Yoshizato $\mathrm{I}$ Yoshida $\mathrm{K}$, et al. Dynamics of clonal evolution in myelodysplastic syndromes. Nat Genet. 2017; 49(2):204-212.

16. Kim T, Tyndel MS, Kim HJ, et al. The clonal origins of leukemic progression of myelodysplasia. Leukemia. 2017;31(9):1928-1935.

17. Stosch JM, Heumuller A, Niemoller C, et al. Gene mutations and clonal architecture in myelodysplastic syndromes and changes upon progression to acute myeloid leukaemia and under treatment. $\mathrm{Br} \mathrm{J}$ Haematol. 2018;182(6):830-842.

18. Ibanez M, Carbonell-Caballero J, Such E, et al. The modular network structure of the mutational landscape of acute myeloid leukemia. PLoS One. 2018;13(10):e0202926.

19. Tamborero D, Rubio-Perez C, Deu-Pons J, et al. Cancer Genome Interpreter annotates the biological and clinical relevance of tumor alterations. Genome Med. 2018;10(1):25.

20. Reiter JG, Baretti M, Gerold JM, et al. An analysis of genetic heterogeneity in untreated cancers. Nat Rev Cancer. 2019;19(11):639650.

21. Thota S, Viny AD, Makishima $\mathrm{H}$, et al Genetic alterations of the cohesin complex genes in myeloid malignancies. Blood. 2014;124(11):1790-1798.
22. Viny AD, Levine RL. Cohesin mutations in myeloid malignancies made simple. Curr Opin Hematol. 2018;25(2):61-66.

23. Pellagatti A, Roy S, Di Genua C, et al. Targeted resequencing analysis of 31 genes commonly mutated in myeloid disorders in serial samples from myelodysplastic syndrome patients showing disease progression. Leukemia. 2016;30(1):247-250.

24. Takahashi K, Jabbour E, Wang $X$, et al. Dynamic acquisition of FLT3 or RAS alterations drive a subset of patients with lower risk MDS to secondary AML. Leukemia. 2013;27(10):2081-2083.

25. Meggendorfer $M$, de Albuquerque $A$, Nadarajah N, et al. Karyotype evolution and acquisition of FLT3 or RAS pathway alterations drive progression of myelodysplastic syndrome to acute myeloid leukemia. Haematologica. 2015;100(12):e487-490.

26. Badar T, Patel KP, Thompson PA, et al. Detectable FLT3-ITD or RAS mutation at the time of transformation from MDS to AML predicts for very poor outcomes. Leuk Res. 2015;39(12):1367-1374.

27. Xu F, Han R, Zhang J, et al. The Role of FLT3 ITD Mutation on de novo MDS in Chinese population. Clin Lymphoma Myeloma Leuk. 2019;19(2):e107-e115.

28. Walter MJ, Shen D, Shao J, et al. Clonal diversity of recurrently mutated genes in myelodysplastic syndromes. Leukemia. 2013;27(6):1275-1282.

29. Miller CA, McMichael J, Dang HX et al. Visualizing tumor evolution with the fishplot package for R. BMC Genomics. 2016; 17(1):880.

30. Mazumdar C, Shen Y, Xavy S, et al Leukemia-associated cohesin mutants dominantly enforce stem cell programs and impair human hematopoietic Progenitor differentiation. Cell Stem Cell. 2015; 17(6):675-688.

31. Mondal G, Stevers M, Goode B, Ashworth A Solomon DA. A requirement for STAG2 in 
replication fork progression creates a targetable synthetic lethality in cohesin-mutant cancers. Nat Commun. 2019;10(1):1686.

32. Meisenberg C, Pinder SI, Hopkins SR, et al. repression of transcription at DNA breaks requires cohesin throughout interphase and prevents genome instability. Mol Cell. 2019; 73(2):212-223.e7.

33. Kuendgen A, Muller-Thomas C, Lauseker M, et al. Efficacy of azacitidine is independent of molecular and clinical characteristics - an analysis of 128 patients with myelodysplastic syndromes or acute myeloid leukemia and a review of the literature. Oncotarget. 2018; 9(45):27882-27894.

34. Polgarova K, Vargova K, Kulvait V, et al. Somatic mutation dynamics in MDS patients treated with azacitidine indicate clonal selection in patients-responders. Oncotarget. 2017;8(67):111966-111978.

35. Tobiasson M, McLornan DP, Karimi M, et al. Mutations in histone modulators are associated with prolonged survival during azacitidine therapy. Oncotarget. 2016;7(16):2210322115.

36. Cabezon M, Bargay J, Xicoy B, et al. Impact of mutational studies on the diagnosis and the outcome of high-risk myelodysplastic syndromes and secondary acute myeloid leukemia patients treated with 5 -azacytidine. Oncotarget. 2018;9(27):19342-19355.

37. Dohner H, Dolnik A, Tang L, et al. Cytogenetics and gene mutations influence survival in older patients with acute myeloid leukemia treated with azacitidine or conventional care. Leukemia. 2018, 32(12):2546-2557. 